\title{
First Trimester Diagnosis of Sirenomelia
}

\author{
Amir Irfan Kazi ${ }^{1,2}$ (1) $\cdot$ Yasmin Iqbal $^{2,3} \cdot$ Sana Elham Kazi ${ }^{2}$
}

Received: 6 September 2015 / Accepted: 22 November 2015/Published online: 30 November 2015

(C) Society of Fetal Medicine 2015

\begin{abstract}
Sirenomelia is a rare congenital anomaly characterized by a single lower extremity which is associated with abnormalities in other organ systems, commonly affecting the gastrointestinal and the urogenital systems. It is sporadic with no increased risk in subsequent pregnancies. In almost all the cases of sirenomelia, a single umbilical artery (SUA) is present which arises from the abdominal aorta. Sirenomelia can be confidently diagnosed in the first trimester while the diagnosis in the second and third trimesters is rendered difficult due to the lack of amniotic fluid in the later gestation. Transvaginal ultrasound and color Doppler have a key role in making the diagnosis and 3D ultrasound helps in better demonstrating the abnormality.
\end{abstract}

Keywords Sirenomelia - Ultrasound · First trimester diagnosis $\cdot$ Single umbilical artery $\cdot$ Severe oligohydramnios

\section{Introduction}

Sirenomelia is a rare congenital anomaly characterized by a single lower extremity and is usually lethal. The name is derived from the Greek mythological creatures 'sirens',

Amir Irfan Kazi

kaziamirirfan@gmail.com

1 Department of Radiology, The Muslim Maternity and General Hospital, Hyderabad, India

2 Centre for Obstetrics, Gynaecology and Infertility, 16-8-646, New Malakpet, Hyderabad, Telangana 500024, India

3 Department of Obstetrics and Gynaecology, The Muslim Maternity and General Hospital, Hyderabad, India which were believed to have a single lower extremity in the form of tail fin of a fish. This single lower extremity shows a wide spectrum of anatomical variants depending on the degree of fusion of bony and soft tissue structures. In addition, the condition is associated with abnormalities in other organ systems, most frequently affecting the gastrointestinal and the urogenital systems.

Stocker and Heifetz classified sirenomelia into seven sub-types based on the phenotype (Fig. 1). In the mildest form Type I, the bony structures are all normally present with only soft tissue fusion. Type VII is the severest form with the presence of only a single lower extremity bone [1]. Previously it was divided into three sub-types based on the appearance of the feet (Fig. 1). In sympus dipus (symmelia), both the feet are seen separately. In sympus monopus (uromelia), a single foot is present. In sympus apus (sirenomelia,) the foot is absent [2]. Due to abnormal fusion, there is nonrotation of the embryological lower limb bud, which normally rotates medially during embryogenesis. As a result, the fibulae, when present, lie medial to the tibiae [3].

\section{Report of Case}

A 21-year-old primigravida with spontaneous conception presented for 11-13 weeks scan. The lady had a nonconsanguineous marriage with no history of known genetic disorders in the family of the couple. On ultrasound examination, a single live fetus with size-date discrepancy showing crown rump length of $59 \mathrm{~mm}$ corresponding to 12 weeks 2 days, was seen. Oligohydramnios was seen. However there was no anhydramnios (which sets in during the later period of gestation). The nuchal translucency measured $1.6 \mathrm{~mm}$, nasal bone was visualized and pre- 


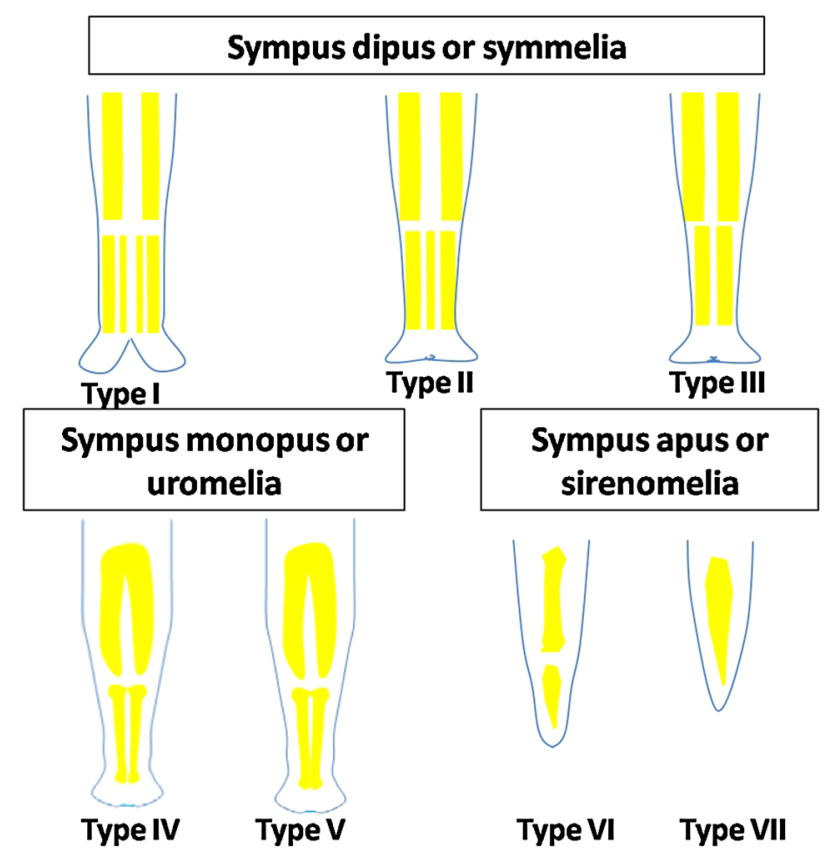

Fig. 1 Stocker and Heifetz classification of Sirenomelia into seven types

maxillary triangle was normal. There was no tricuspid regurgitation. Ductus venosus showed normal spectral pattern. Single femur was visualized with a shortened single bone in the distal lower extremity (Fig. 2a). Both the upper extremities were normal. On color Doppler imaging, a single large intra-abdominal blood vessel was shown, which continued anteriorly and into the two-vessel umbilical cord (Fig. 2b, c). The fetal left kidney was visualized which was hydronephrotic with thinning of the cortex (Fig. 2d). The right kidney and fetal bladder were not visualized. Based on these findings, a diagnosis of sirenomelia (Stocker and Heifetz Type VI) was made. Because of the early gestational age, other associated anomalies could not be demonstrated.

The couple were informed of the findings and received genetic counseling. They opted not to undergo genetic testing and continued the pregnancy. However, they opted for termination of pregnancy at 19 weeks. The antenatal findings were confirmed on delivery (Fig. 2e). In addition, there was absence of external genitalia and anal atresia. The couple did not agree for fetal autopsy.

\section{Discussion}

Sirenomelia is reported to have an incidence of one in 60,000 to one in 100,000 births and has been reported in all ethnic groups across the globe [3]. Males are more commonly affected with a sex ratio of 2.7:1 [4]. It is much

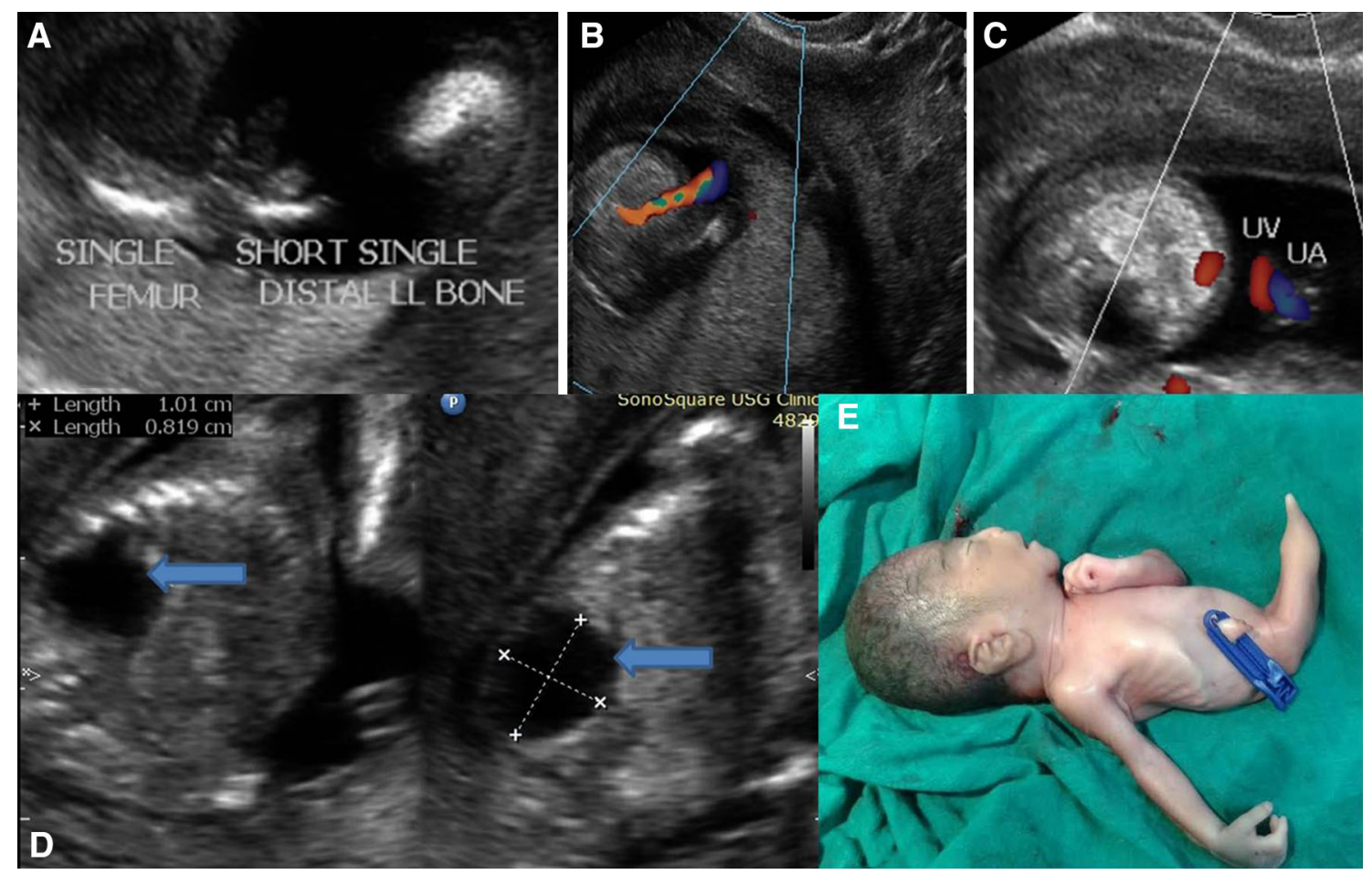

Fig. 2 a A single lower extremity is seen showing a femur and a single distal long bone with absence of foot. b A single umbilical artery seen arising from abdominal aorta. c Two vessel cord. d Hydronephrotic fetal kidney in axial and longitudinal planes showing thinning of renal cortex. e Stillborn fetus showing sirenomelia (Stocker Type VI) 
more common in monozygotic twins with 100 - to 150 -fold increase compared with singleton pregnancy [5]. It is a sporadic condition and the fetal karyotyping is usually normal $[3,6]$. There is no increased risk of recurrence in subsequent pregnancies [7]. In almost all the cases of sirenomelia, a single umbilical artery (SUA) is present. This artery shows an aberrant origin, arising directly from the abdominal aorta high up in the abdomen, usually below the origin of celiac artery. This aberrant SUA is believed to be derived from the embryonic vitelline artery complex and is also known as persistent vitelline artery. Below the level of this aberrant artery, the aorta shows markedly decreased caliber, with paucity of branches supplying the lower extremities, large intestine, and the urogenital tract [3].

While the primary mechanism leading to sirenomelia remains unknown, many theories have been proposed to explain the causal mechanisms. The two important theories are the vascular steal hypothesis and the defective blastogenesis hypothesis. The vascular steal hypothesis is based on the abnormal vascular distribution of the distal body seen in most fetuses having sirenomelia. This hypothesis proposes that the SUA of vitelline origin diverts blood flow to the placenta, leading to the lower part of the body having markedly reduced blood supply and consequently, the organs receiving their blood supply from these vessels may not develop, may show malformation, or may be incompletely developed [8]. The presence or absence of kidneys depends on whether this artery arises above or below the level of renal arteries [7]. Nevertheless, there is no conclusive evidence to categorically establish vascular abnormality as the etiology, and the probability of an earlier insult in the embryological development cannot be excluded [3].

According to the defective blastogenesis hypothesis, sirenomelia is believed to be secondary to the maldevelopment of the caudal somites and tailbud, a process which occurs in the final stages of gastrulation at about the third week of gestation in humans [1,9]. The anatomical difference depends on the intensity, time of initiation, and duration of the underlying event. The proponents of this theory believe that sirenomelia is a variant of caudal regression syndrome (caudal dysgenesis). However, sirenomelia presents with features that are different from caudal dysgenesis including the presence of SUA of vitelline origin, cases not having dorsal abnormalities of the neural tube and spine, and no obvious association with maternal diabetes mellitus [3]. In addition, in caudal regression syndrome, the lower limbs are nonfused and liquor is usually adequate [6, 7].

Almost all the cases of sirenomelia show renal and urethral abnormalities. Frequently, there is bilateral renal agenesis leading to severe oligohydramnios with absent liquor [3]. Multicystic dysplastic kidneys may also be present [7]. Exceptional cases of neonates with minor renal abnormalities or even normal kidneys have been reported [4]. The urinary tract anomalies are associated with maldevelopment of the genital tract, predominantly affecting the external genitalia. The external genitalia may be absent or may even be represented by a tag of tissue [3]. Ambiguous genitalia may also be seen. Mullerian anomalies may be present [7]. The gonads are usually unaffected [10]. The abnormalities of the distal gastrointestinal tract are also frequent, the most common being anorectal atresia. Malformations in the lumbosacral spine, hypoplasia/aplasia of pelvic girdle may be seen. Less commonly, cardiac or central nervous system abnormalities may be seen. Differential diagnosis for sirenomelia includes bilateral renal agenesis (Potter syndrome), renal dysplasia, obstructive uropathy, and also severe fetal growth restriction. The diagnosis is not easy in second and third trimesters due to severe oligohydramnios caused by absent or nonfunctioning kidneys [6, 7].

Very few cases of first trimester diagnosis of sirenomelia have been reported [6]. The anomaly can be confidently diagnosed in the first trimester on ultrasonography. During the late second and third trimesters, anhydramnios may limit the diagnosis of this condition. With the help of transvaginal ultrasonography, all the long bones of the fetus can be visualized at 10 weeks of gestation [5]. During the first trimester, liquor is produced predominantly by the lining of the amniotic cavity allowing better evaluation of the fetus. Transvaginal ultrasonography with the use of color Doppler is essential in evaluating the anatomy and the associated vascular maldevelopment. 3D ultrasonography, though not essential, helps in better depicting the abnormality and increases the diagnostic confidence. MRI has a role in confirming the diagnosis in later gestation where adequate evaluation on ultrasound may not be possible due to absence of liquor.

Sirenomelia is fatal in most cases secondary to the pulmonary hypoplasia caused by the severe oligohydramnios due to renal agenesis. Very few cases have been reported where the fetuses survived the neonatal period. Postnatal survival in the cases of sirenomelia is possible only with the presence of a functioning renal system without total obstructive renal failure, which can be assessed antenatally by the adequacy of the amniotic fluid. The babies who have survived have required extensive surgeries for anorectal and genitourinary abnormalities and limb reconstruction $[11,12]$.

\section{Conclusion}

Sirenomelia is a rare congenital anomaly which carries a poor prognosis. It is sporadic in occurrence. It can be confidently diagnosed in the first trimester while the 
diagnosis in the second and third trimesters may be difficult due to the lack of amniotic fluid in later gestation. Transvaginal ultrasonography and color Doppler have a key role in making the diagnosis and 3D ultrasound helps in better characterization of the abnormality.

\section{Compliance with ethical standards}

Conflict of interest None.

\section{References}

1. Stocker JT, Heifetz SA. Sirenomelia. A morphological study of 33 cases and review of literature. Perspect Pediatr Pathol. 1987;10:7-50.

2. Taori KB, Mitra K, Ghonga NP, Gandhi RO, Mammen T, Sahu J. Sirenomelia sequence (mermaid): report of three cases. Indian $\mathbf{J}$ Radiol Imaging. 2002;12:399-401.

3. Garrido-Allepuz C, Haro E, González-Lamuño D, Martínez-Frías ML, Bertocchini F, Ros MA. A clinical and experimental overview of sirenomelia: insight into the mechanisms of congenital limb malformations. Dis Models Mech. 2011;4:289-99.

4. Valenzano M, Paoletti R, Rossi A, Farinini D, Garlaschi G, Fulcheri E. Sirenomelia. Pathological features, antenatal ultrasonographic clues and a review of current literature. Hum Reprod Update. 1999;5(1):82-6.
5. Monteagudo A, Mayberry P, Rebarber A, Paidas M, TimorTritsch IE. Sirenomelia sequence first-trimester diagnosis with both two- and three-dimensional sonography. J Ultrasound Med. 2002;21:915-20.

6. Solovyov O, Goncharova Y, Masliy Y, Zukin V, Sudoma I. Sirenomelia with urethral atresia, corresponding megacystis and bilateral hydronephrosis, diagnosed in the first trimester of pregnancy after assisted reproductive technologies. Arch Perinat Med. 2009;15(3):136-43.

7. Byrne JLB. Sirenomelia. In: Woodward PJ, Kennedy A, Sohaey R, et al., editors. Diagnostic imaging obstetrics. 1st ed. Salt Lake City: Amirsys; 2005.

8. Stevenson RE, Jones KL, Phelan MC, Jones MC, Barr M Jr, Clericuzio C, Harley RA, Benirschke K. Vascular steal: the pathogenetic mechanism producing sirenomelia and associated defects of the viscera and soft tissues. Pediatrics. 1986;78(3):451-7.

9. Opitz JM, Zanni G, Jr Reynolds J F, Gilbert-Barness E. Defects of blastogenesis. Am J Med Genet. 2002;115:269-86.

10. Kallen B, Castilla EE, Lancaster PA, Mutchinick O, Knudsen LB, Martínez-Frías ML, Mastroiacovo P, Robert E. The cyclops and the mermaid: an epidemiological study of two types of rare malformation. J Med Genet. 1992;29(1):30-5.

11. Pinette MG, Hand M, Hunt RC, Blackstone J, Wax JR, Cartin A. Surviving sirenomelia. J Ultrasound Med. 2005;24:1555-9.

12. Goodman SH, Ko DS, Johnson HW, Coleman GU, Murphy JJ, Fraser GC. Sirenomelia:urological abnormalities in a surviving mermaid. Can J Urol. 1996;3:257-60. 